\title{
Simultaneous and Anonymous Mobile Network Authentication Scheme Based on Chaotic Maps
}

\author{
Wen-Chung Kuo ${ }^{1}$, Chi-Sheng Lin ${ }^{2}$, Chen-Tsun Chuang ${ }^{2}$, Ming-Chih Kao ${ }^{3}$ \\ ${ }^{1}$ National Yunlin University of Science \& Technology, \\ Department of Computer Science and Information Engineering \\ No.123 University Road, Section 3, Douliou, Yunlin 64002, Taiwan, R.O.C \\ e-mail:simonkuo@yuntech.edu.tw \\ ${ }^{2}$ Department of Computer Science and Information Engineering, \\ National Formosa University \\ No.64, Wunhua Rd., Huwei Township, Yunlin County 632, Taiwan, R.O.C. \\ ${ }^{3}$ System Integration Depart, Internet Technology Software Division, \\ Computational Intelligence Technology Center, \\ Industrial Technology Research Institute, Taiwan, R.O.C. \\ crossref http://dx.doi.org/10.5755/j01.itc.45.2.8875
}

\begin{abstract}
Wireless network authentication schemes have been researched for about a decade, and associated security problems explored by many researchers. In 2004, Zhu and Ma proposed an authentication scheme for wireless environments. Afterwards other researchers proposed improvements according to Zhu-Ma scheme. Mutual authentication is one of the most important issues. To the best of our knowledge, there is no scheme that allows a foreign agent to authenticate the home agent and mobile user at the same time. Towards this purpose, we propose a simultaneous authentication scheme based on chaotic maps, and describe how our proposed scheme addresses various security problems while maintain anonymity.
\end{abstract}

Keywords: Authentication; Mutual authentication; Chaotic-maps; security.

\section{Introduction}

Wireless communication is ubiquitous and the number of people who use wireless network services is increasing massively. This leads to situations on the network where a server which offers network service has to authenticate a mobile user $(M U)$ in a wireless environment. However, the security landscapes of wireless networks have been studied for about a decade and research is still maturing.

In 2004, Zhu and Ma [12] proposed an authentication scheme with anonymity for the wireless environment, taking into account the limited network resources and a higher channel error rate in wireless communication environment. There are two major advantages in their scheme: 1) it takes only one round of message exchange between the mobile user and the foreign network, and another round of message exchange between the foreign network and the home network; 2) a one-time key is used between the mobile user and the foreign network. Then, in 2006, Lee et al.
[5] pointed out that there are security weaknesses in the Zhu-Ma scheme such as not achieving mutual authentication or providing backward secrecy of session key. In response, they provided an enhanced protocol (LHL-scheme) to Zhu-Ma's scheme. In 2008, Wu et al. [8] found another security issue that affects the Zhu-Ma and LHL schemes since they only use a one-way hash function to hide the user's real identity. An attacker could easily obtain $I D_{M U}$ by off-line guessing attack. They proposed a modification (WLTscheme) to strengthen the properties of anonymity and backward secrecy. The next year, Lee et al. [7] demonstrated the WLT-scheme still cannot provide the property of anonymity which was an inherited issued from Zhu-Ma scheme. In 2009, Zeng et al. [11] pointed out an inherent design flaw in Zhu-Ma scheme, in which an adversary can register as a legitimate user to the home agent $(H A)$, and obtain the real identity of other users via messages eavesdropped between the foreign agent $(F A)$ and mobile user. In 2010, He et al. [3] proposed a strong user 
authentication scheme (HMZCB-scheme) using smart card for wireless environments. It focuses on the situation of smart card breach, even if the adversary extracts the information stored in smart card, he cannot derive the password of the user. In 2011, Lee and Kwon [6] provided the property of untraceability for the user. They used random nonce to make each session requests from a particular user unidentifiable, so the adversary cannot tell if the message is from the same user or not. To the best of our knowledge, there is no scheme that allows a foreign agent to authenticate the home agent and mobile user at the same time using only one function. Towards this purpose, we propose a simultaneous authentication scheme and describe how our proposed scheme addresses various security problems in this paper.

Recently, cryptosystems [1, 2, 9, 10] based on chaotic-maps theory (nonlinear dynamic) have been studied widely because the operations of chaotic-maps use recurrence characteristics. For example, Chain and Kuo [1] proposed a new identity feature based on the chaotic maps for digital signature scheme. We now extend this identity feature for an anonymous wireless communication authentication scheme. The major contribution of this proposed scheme is authentication of the foreign agent $(F A)$ to the mobile user $(M U)$ and the $F A$ to the home agent $(H A)$, in other words, $F A$ can authenticate $H A$ and $M U$ simultaneously, which is a unique approach not applied in previous schemes.

The rest of our paper is organized as follows: In Section 2, we briefly introduce Chebyshev chaoticmaps and their characteristics. In Section 3, we present our proposed scheme. The analysis of our scheme is presented in Section 4. Concluding remarks are given in Section 5.

\section{Preliminaries}

In this section, first we will briefly introduce Chebyshev chaotic maps and their properties [1].

\subsection{Chebyshev Chaotic Maps}

Let $n$ be an integer and $x$ be a variable within the interval $[-1,1]$. The Chebyshev polynomial $T_{n}(x)$ is defined as:

$$
T_{n}(x)=\cos \left(n \cos ^{-1}(x)\right) .
$$

With Eq.(1), the recurrence relation $T_{n}(x)$ is defined as:

$$
T_{n}(x)=2 x T_{n-1}(x)-T_{n-2}(x),
$$

where $n>=2, T_{0}(x)=1$ and $T_{1}(x)=x$. One of the most important properties of Chebyshev polynomials is the semi-group property which establishes the following:

$$
\begin{aligned}
& T_{r}\left(T_{s}(x)\right)=\cos \left(r \cos ^{-1}\left(\cos \left(s \cos ^{-1}(x)\right)\right)\right) \\
& =\cos \left(r s \cos ^{-1}(x)\right)=T_{s r}(x)=T_{r}\left(T_{s}(x)\right) .
\end{aligned}
$$

\subsection{Chaotic Maps Problems [1]}

Let $P$ and $Q$ be integers and $p$ be a prime number. The general second-order linear recurrence relation is as follows:

$$
T_{n}(M)=P \times T_{n-1}(M)+Q \times T_{n-2}(M) \bmod p,(4)
$$

where $n>=2, T_{n}(M) \in G F(p), T_{0}(M)=1$ and $T_{1}(M)=M$.

Theorem 1: Let $f(M)=t^{2}-2 M t+1$ and $\alpha, \beta$ be the roots of $f(M)$. If $M=\frac{1}{2}(\alpha+\beta)$, then the number of solutions is:

$$
T_{n}(M)=\frac{\left(M+\sqrt{M^{2}-1}\right)^{n}+\left(M-\sqrt{M^{2}-1}\right)^{n}}{2} \bmod p .
$$

Theorem 2: If $a$ and $b$ are integers, and $a>b(a, b \in$ $\left.Z^{+}\right)$, then

$$
T_{a+b}(M)+T_{a-b}(M)=2 T_{a}(M) T_{b}(M) .
$$

Theorem 3: If $a=b+c$, then

$$
\begin{aligned}
& \left(T_{a}(M) T_{b}(M) T_{a}(M)+1\right) \bmod p=\left(\left[T_{a}(M)\right]^{2}+\right. \\
& \left.\left[T_{b}(M)\right]^{2}+\left[T_{c}(M)\right]^{2}\right) \bmod p .
\end{aligned}
$$

\section{The Proposed Scheme}

In this section, we will introduce our proposed scheme in detail. Our scheme is composed of the following three phases: Registration phase, Authentication phase, and Session key update phase. Before we introduce our proposed scheme, the notations and symbols used in the proposed scheme are as shown Table 1.

Table 1. Notations

\begin{tabular}{cc}
\hline Symbol & Represents \\
\hline$M U$ & The mobile user \\
$F A$ & The foreign agent \\
$H A$ & The home agent \\
$P M_{M U}$ & The password of mobile user \\
$I D_{A}$ & The identity of an entity $A$ \\
$h(\cdot)$ & A collision free one-way hash function \\
$p_{M U}$ & The secret key selected by $M U$ \\
$\oplus$ & The exclusive-OR operation \\
$\|$ & The concatenation operation \\
$N_{A}$ & A random nonce selected by an entity $A$ \\
$P$ & A point on the elliptic curve $E_{p}(a, b)$ \\
\hline
\end{tabular}

\subsection{Registration Phase}

As shown in Fig. 1, $M U$ selects a secret key $S$, and computes $P W_{M U}=h\left(I D_{M U} \| s\right)$. Then $M U$ sends $I D_{M U}$ and $P W_{M U}$ to $H A$ via a secure channel. When 
MU

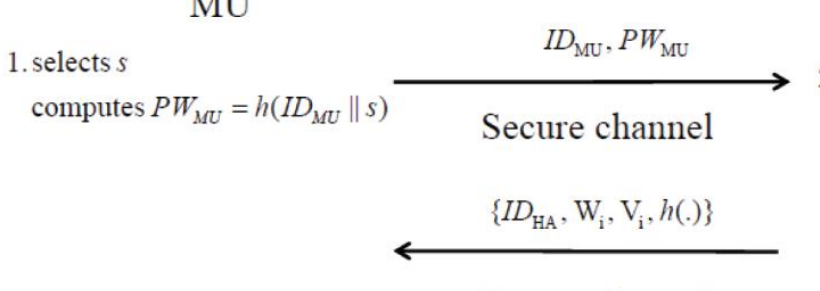

Secure channel
HA

2.generates $\mathrm{N}_{\mathrm{MU}_{\mathrm{i}}}$ and $s_{\mathrm{HA}-\mathrm{MU}_{\mathrm{i}}}$ computes $\mathrm{U}=h\left(s_{\mathrm{HA}-\mathrm{MU}_{\mathrm{i}}} \| \mathrm{N}_{\mathrm{MU}_{\mathrm{i}}}\right)$

$$
\mathrm{W}_{\mathrm{i}}=P W_{\mathrm{MU}} \oplus \mathrm{N}_{\mathrm{MU}_{\mathrm{i}}}
$$$$
\mathrm{V}_{\mathrm{i}}=\mathrm{N}_{\mathrm{MU}_{\mathrm{i}}} \oplus s_{\mathrm{HA}-\mathrm{MU}}
$$

stores $P W_{\mathrm{MU}}, s_{\mathrm{HA}-\mathrm{MU}}$ in database

Figure 1. The Registration phase

$H A$ receives the message, it generates random nonce $N_{M U_{i}}$ and $S_{H A-M U_{i}}$, and then computes $U=$ $h\left(s_{H A-M U_{i}} \| N_{M U_{i}}\right), W_{i}=P W_{M U} \oplus N_{M U_{i}}$ and $V_{i}=$ $N_{M U_{i}} \oplus s_{H A-M U_{i}} . H A$ stores $P W_{M U}$ and $s_{H A-M U_{i}}$ into its database and uses $U$ as an entry of $M U$. Then $H A$ stores $I D_{M U}, W_{i}, V_{i}, h(\cdot)$ in the smart card and sends the smart card to $M U$ via secure channel.

\subsection{Authentication phase}

Step A1: $M U$ computes $P W_{M U}=h\left(I D_{M U} \| s\right)$.

Step A2: $M U \rightarrow F A: L_{1}=\left\{I D_{H A}, M_{1}, M_{2}, M_{3}, M_{4}\right\}$

After user verification, $M U$ generates a random nonce $N_{M U_{i+1}}$ and extracts $N_{M U_{i}}$ and $S_{H A-M U_{i}}$ from $N_{M U_{i}}=P W_{M U} \oplus W_{i} \quad$ and $\quad s_{H A-M U_{i}}=N_{M U_{i}} \oplus V_{i}$. Then $M U$ computes $M_{1}=h\left(s_{H A-M U_{i}} \| N_{M U_{i}}\right), M_{2}=$ $P W_{M U} \oplus N_{M U_{i+1}}, \quad M_{3}=h\left(N_{M U_{i+1}} \| I D_{F A}\right), \quad M_{4}=$ $h\left(P W_{M U} \oplus h\left(s_{H A-M U_{i}} \| N_{M U_{i+1}}\right)\right)$, and attaches $I D_{H A}$ to form $L_{1}$. The $M U$ sends the authentication message $L_{1}$ to $F A$.

Step A3: $F A \rightarrow H A: L_{2}=\left\{I D_{F A}, M_{1}, M_{2}, M_{5}, M_{6}\right\}$

$F A$ first generates three variables $a, b, c$, where $c=a+b$. Next, $F A$ computes $M_{5}=M_{3} \oplus M_{4}$ and $M_{6}=M_{5} \oplus a$, then $F A$ stores $I D_{H A}$ and sends message $L_{2}$ to $H A$.

Step A4: $H A \rightarrow F A: L_{3}=\left\{I D_{H A}, M_{8}, M_{9}, M_{10}\right\}$

$H A$ uses $M_{1}$ as an entry to extract $P W_{M U}$ and $S_{H A-M U_{i}}$ stored in its database. Then $H A$ computes $N_{M U_{i+1}}^{\prime}=P W_{M U} \oplus M_{2}, M_{3}^{\prime}=h\left(N_{M U_{i+1}}^{\prime} \| I D_{F A}\right)$, $M_{4}^{\prime}=h\left(P W_{M U} \oplus H\left(s_{H A-M U_{i}} \| N_{M U_{i+1}}^{\prime}\right)\right), \quad M_{5}^{\prime}=$ $M_{3}^{\prime} \oplus M_{4}^{\prime}$, and checks if $M_{5}^{\prime}$ equals $M_{5}$. If it is true, $H A$ computes $a=M_{6} \oplus M_{5}^{\prime}, \quad M_{7}=h\left(P W_{M U} \|\right.$ $\left.N_{M U_{i+1}}^{\prime}\right), M_{8}=h\left(I D_{F A}\left\|I D_{H A}\right\| M_{7}\right), M_{9}=T_{a}\left(M_{3}\right)$, $M_{10}=h\left(M_{9} \| M_{7}\right)$. Finally, $H A$ sends the message $L_{3}$ to $F A$ and replaces $M_{1}$ with $h\left(s_{H A-M U_{i}} \| N_{M U_{i+1}}\right)$ as a new entry of $M U$.

Step A5: $F A \rightarrow M U: L_{4}=\left\{I D_{F A}, M_{8}, M_{9}, M_{11}, M_{12}\right\}$

After receiving message $L_{3}, F A$ verifies the identity of $H A$. If it is verified, $F A$ computes $M_{11}=$ $b \oplus M_{10}, M_{12}=T_{c}\left(M_{3}\right)$, and then sends message $L_{4}$ to $M U$. $M U$ receives message $L_{4}$ and computes $M_{7}^{\prime}=$
$h\left(P W_{M U} \| N_{M U_{i+1}}\right) \quad$ and $\quad M_{8}^{\prime}=h\left(I D_{F A}\left\|I D_{H A}\right\|\right.$ $\left.h\left(P W_{M U} \| N_{M U_{i+1}}\right)\right)$ and verifies if $M_{8}^{\prime}=M_{8}$.

Step A6: $M U \rightarrow F A: L_{5}=\left\{M_{13}, M_{14}, C_{M F}\right\}$

If the verification holds, $M U$ computes $b=M_{11} \oplus$ $h\left(M_{9} \oplus M_{7}^{\prime}\right), M_{13}=T_{b}\left(M_{3}\right)$ and selects a variable $d$ then computes $M_{14}=T_{d}\left(M_{3}\right)$, the session key $h\left(T_{d c}\left(M_{3}\right)\right)$ and $C_{M F}=h\left(T_{d c}\left(M_{3}\right) \| b\right)$. Then $M U$ sends message $L_{5}$ to $F A$.

After receiving message $L_{5}$ sent from $M U, F A$ verifies if $M_{13}^{2}+T_{c}^{2}\left(M_{3}\right)+M_{9}^{2}=M_{13} \times T_{c}\left(M_{3}\right) \times$ $M_{9}+1$. If it holds, $F A$ then computes the session key $K_{M F}^{\prime}=T_{c}\left(M_{14}\right)=T_{c d}\left(M_{3}\right)$ and checks whether $C_{M F}$ is equal to $h\left(K_{M F}^{\prime} \| b\right)$ or not. If it holds, then the session key between $F A$ and $M U$ is $T_{c d}\left(M_{3}\right)$; otherwise $F A$ rejects the $M U$ 's request. The detailed process is shown in Fig. 2.

\subsection{Session key update phase}

Step S1: $M U$ first selects $d_{i}$, and computes $T_{d_{i}}\left(M_{3}\right)$, then sends $\left\{T_{d_{i}}\left(M_{3}\right), C_{M F_{i-1}}\right\}$ to $F A$.

Step S2: After receiving the message, $F A$ extracts $T_{c_{i-1}}$ by $C_{M F_{i-1}}$ from its database. $F A$ computes $\quad K_{M F_{i}}=T_{c_{i} d_{i}}\left(M_{3}\right) \quad, \quad C_{M F_{i}}=$ $h\left(K_{M F_{i}} \| T_{d_{i}}\left(M_{3}\right)\right)$, and calculates $h_{1}=$ $h\left(C_{M F_{i}} \| T_{C_{i-1}}\left(M_{3}\right)\right)$. Then $F A$ sends $\left\{T_{c_{i}}\left(M_{3}\right), h_{1}\right\}$ to $M U$ and replaces $T_{c_{i-1}}\left(M_{3}\right)$, $C_{M F_{i-1}}$ with $T_{c_{i}}\left(M_{3}\right)$ and $C_{M F_{i}}$.

Step S3: $M U$ computes $K_{M F_{i}}=T_{d_{i} c_{i}}\left(M_{3}\right), C_{M F_{i}}=$ $h\left(K_{M F_{i}} \| T_{d_{i}}\left(M_{3}\right)\right)$ and $h_{1}^{\prime}=h\left(C_{M F_{i}} \|\right.$ $\left.T_{c_{i-1}}\left(M_{3}\right)\right)$, and $M U$ verifies if $h_{1}^{\prime}=h_{1}$. If true, $M U$ stores $T_{c_{i}}\left(M_{3}\right)$ and $C_{M F_{i}}$.

\subsection{Modify Password Phase}

Generally, $M U$ can safely modify his password with $H A$ through public channels. The procedure of the password change phase is described as follows.

Step M1: $M U \rightarrow H A: U, h_{\text {new } 1}, h_{\text {new } 2}$

$M U$ selects a new random number $s_{\text {new }}$ and computes $P W_{M U_{\text {new }}}=h\left(I D_{M U} \| s_{\text {new }}\right) \quad, \quad U=$ $h\left(s_{H A-M U_{i}} \| N_{M U_{i}}\right), h_{\text {new } 1}=P W_{M U} \oplus P W_{M U_{\text {new }}}$ and 


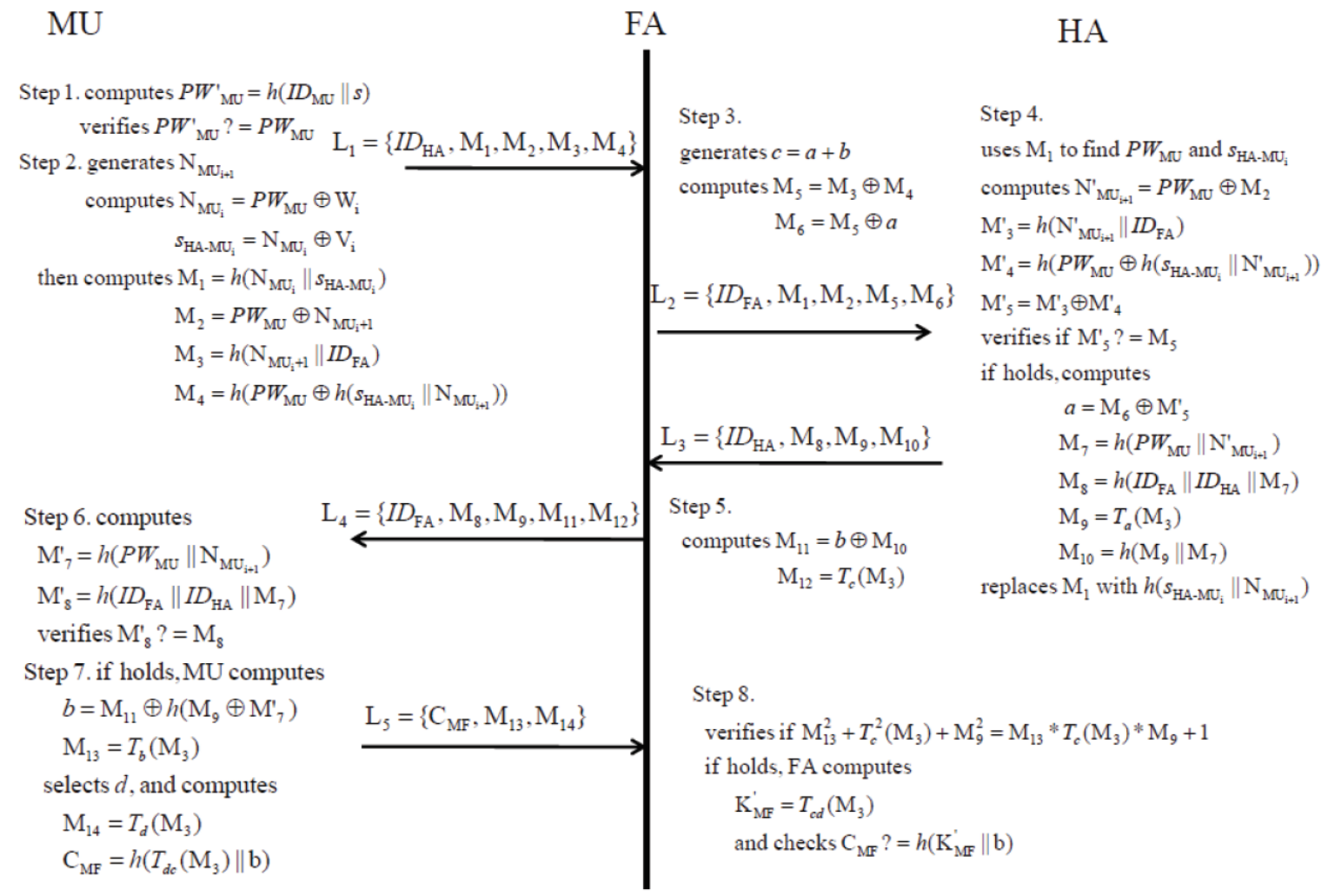

Figure 2. The Authentication phase

$h_{\text {new } 2}=h\left(P W_{M U_{\text {new }}} \| s_{H A-M U_{i}}\right)$. Then, $M U$ sends $U, h_{\text {new } 1}$, and $h_{\text {new } 2}$ to $H A$.

Step M2:HA $\rightarrow M U: h_{\text {new3 }}$

$H A$ extracts the corresponding $P W_{M U}$ and $S_{H A-M U_{i}}$ from its database using $U$ and calculates $P W_{M U_{n e w}}^{\prime}=$ $P W_{M U} \oplus h_{n e w 1} \quad$ and $\quad h_{n e w 2}^{\prime}=h\left(P W_{M U_{n e w}}^{\prime} \|\right.$ $\left.S_{H A-M U_{i}}\right)$. If $h_{\text {new2 }}$ is equal to $h_{\text {new } 2}^{\prime}$, then $H A$ can compute $h_{\text {new } 3}=h\left(P W_{M U} \| S_{H A-M U_{i}}\right)$ and replace $P W_{M U}$ with $P W_{M U_{\text {new }}}$. $H A$ also sends $h_{\text {new } 3}$ to $M U$. Otherwise, $H A$ stops this password modification request.

Step M3: $M U \quad$ calculates $\quad h_{\text {new } 3}^{\prime}=h\left(P W_{M U} \|\right.$ $\left.S_{H A-M U_{i}}\right)$ and checks whether $h_{\text {new } 3}$ is equal to $h_{\text {new } 3}^{\prime}$. If so, then $M U$ updates $W_{i}$ with $P W_{M U_{\text {new }}} \oplus N_{M U_{i}}$. Otherwise, $M U$ rejects this modified option.

\section{Security analysis}

\subsection{Anonymity}

In our proposed scheme, the identity of $M U$ is contained in these messages: $P W_{M U}, M_{2}, M_{4}, M_{5}, M_{6}$, $M_{8}$ and $M_{10}$. We assume that the adversary can intercept these messages and try to extract the identity of $M U$. In this scenario, without knowing $N_{M U_{i+1}}$ and $S_{H A-M U_{i}}$, the adversary cannot calculate the identity of $M U$. Some of the messages are protected by one-way hash which increases computation for guessing-attack. As a result, our proposed scheme can effectively keep anonymity.

\subsection{Man in the middle attack}

The man-in-the-middle attack (abbreviated as MITM) is a kind of wireless network attack via connection eavesdropping, impersonation or both. We assume the channel is eavesdropped bidirectionally by the adversary between $M U$ and $F A$, i.e. the adversary can receive messages $L_{1}, L_{4}$ and $L_{5}$. However, the adversary doesn't know $N_{M U_{i+1}}$ and $P W_{M U}$, and we use hash to process sensitive information. So the password of the user would be safe since the adversary cannot extract it from those messages.

\subsection{Secrecy of the session key}

In our proposed scheme, $M U$ and $F A$, respectively, use $T_{d}\left(M_{3}\right)$ and $T_{c}\left(M_{3}\right)$ as public keys, and compute $K_{M F}=T_{d c}\left(M_{3}\right)$ as their session key. We assume the adversary can intercept both public keys and try to calculate the session key. Since $d$ and $c$ are secret variables only known by $M U$ and $F A$, respectively, it is very difficult to calculate the session key. In the session key update phase, our scheme changes $d$ and $c$ for each update phase process. The adversary still cannot compute the session key correctly. Hence, our scheme keeps the secrecy of the session key. 


\subsection{Replay attack}

Each time $M U$ sends an authentication request, $M U$ will generate a new random nonce $N_{M U_{i+1}}$ to compute the request message. When receiving the request message, $H A$ will use $M_{1}$ to find the corresponding entry of the user in its database. After successful authentication, $H A$ will replace $M_{1}$ with a new entry computed with $N_{M U_{i+1}}$. If an adversary intercepts the authentication request and tries to resend it, $H A$ will recognize this message as a replayed message and reject it. In the session key update phase, $M U$ generates a new $d_{i}$ to compute $T_{d_{i}}\left(M_{3}\right)$ each time, and $F A$ uses $C_{M F_{i}}$ to find the entry of the user in its database. After every successful update, $F A$ will replace $C_{M F_{i}}$ with $C_{M F_{i+1}}$. If an adversary intercepts the message and resents it, $F A$ will recognize this message as a replay message and reject it. As a result, our proposed scheme can prevent replay attack.

\subsection{Untraceability}

To achieve this property, we have to achieve two things: 1.the identity of user needs to be hidden; 2.the temporary identity of user needs to be changed at each session. In our proposed scheme, we issue a temporary identity instead of the real identity for $M U$ during authentication. For each session, $M U$ generates a new random nonce $N_{M U_{i+1}}$ to compute the authentication request message. Since the message changes at every session, even if the request message is intercepted, an adversary discerns if the message is sent from a particular $M U$. Meanwhile, our proposed scheme keeps users untraceable.

\subsection{Mutual authentication}

This subsection discusses mutual authentication between the three parties of $M U, F A$ and $H A$. First, for mutual authentication between $M U$ and $H A, H A$ authenticates $M U$ by verifying $M_{5}$ in Step A4, and $H A$ uses $N_{M U_{i+1}}$ and $P W_{M U}$ to compute $M_{8}$, then $M U$ computes $M_{8}^{\prime}$ to authenticate $F A$ in Step A6. Next, for authentication from $M U$ and $H A$ to $F A$. $M U$ authenticates $F A$ by verifying $M_{8}$, and $H A$ can authenticate $F A$ by verifying $M_{5}$.

\subsection{Simultaneous authentication}

The proposed scheme provides the special property of simultaneous authentication. In other words, the foreign agent $F A$ is able to authenticate the home agent $H A$ and the mobile user $M U$ at the same time by using one function. The following explains how simultaneous authentication occurs. In Step A3, FA generates $c=a+b$, and sends $a$ and $b$ attached to the computed messages to $H A$ and $M U$, respectively. Then $H A$ and $M U$ sends $T_{a}\left(M_{3}\right), T_{b}\left(M_{3}\right)$ back. We apply the identity property of chaotic maps, where $T_{a}^{2}\left(M_{3}\right)+T_{b}^{2}\left(M_{3}\right)+T_{c}^{2}\left(M_{3}\right)=T_{a}\left(M_{3}\right) T_{b}\left(M_{3}\right) T_{c}$ $\left(M_{3}\right)+1$. Hence, $F A$ verifies $H A$ and $M U$ as $M_{13}^{2}+$
$T_{c}^{2}\left(M_{3}\right)+M_{9}^{2}=M_{13} T_{c}\left(M_{3}\right) M_{9}+1$. If the equation holds, FA successfully authenticates $H A$ and $M U$ simultaneously. Therefore, our proposed scheme has 3-party simultaneous authentication for the $F A$ 's side, in which if an adversary intercepts the message between $M U$ and $F A$ to get the value $b$ from $M_{11}=$ $b \oplus M_{10}$, he/she still does not know $M_{10}$ which is computed in hash-function. Accordingly, our scheme can provide secure and thorough authentication.

\subsection{Smartcard loss attack}

In our proposed scheme, even if an attacker obtains a legitimate user's smartcard, he only can extract $I D_{H A}, W_{i}, V_{i}$ and $h(\cdot)$. However, the legitimate user's $P W_{M U}$ is not stored in this smartcard directly. So, the password $P W_{M U}$ is protected because $P W_{M U}$ is included in the $W_{i}$ and calculated with $N_{M U_{i}}$. It is not feasible to obtain the $P W_{M U}$ from $W_{i}$. Therefore, our proposed scheme prevents unauthorized use of lost smartcards.

Finally, we compare our proposed scheme with previous schemes [3, 4, 5, 6, 8, 12] in terms of security and show the comparison results in Table6(')@. According to Table 6(')@, there are six major characteristics:

1. User anonymity. User anonymity is not established in schemes $[3,5,8,12]$. An attacker can obtain a user's real identity by intercepting packets.

2. The schemes in $[5,8,12]$ cannot prevent impersonation or man-in-the-middle attack.

3. Session key secrecy. The schemes $[5,12]$ do not provide secrecy of the session key. The other schemes, including ours, do provide session key protection.

4. Untraceability. Both our scheme and LeeKwon's scheme can achieve untraceability and prevent the disclosure of the user's whereabouts. They both generate a different random nonce for each authentication phase.

5. Lost smartcard protection. Similar to [4], our proposed scheme prevents smartcard loss attack because the $P W_{M U}$ is not saved on the smart card, i.e., the attacker cannot obtain user's $P W_{M U}$ from smart card when it is lost/stolen.

6. Authentication. In the KWC scheme[4], the validation of $I D_{H A}$ is used for mutual authentication between $F A$ and $H A$. In comparison, the proposed method authenticates $F A$ with $H A$ and $M U$ simultaneously.

\section{Conclusion}

In this paper, we proposed a new capability for a wireless communication authentication scheme which allows $F A$ to simultaneously authenticate $M U$ and $H A$. In addition, we also show the proof of resistance 
against various security attacks while maintaining anonymity.

\section{Acknowledgement}

This work was supported in part by the Ministry of Science and Technology of the Republic of China under Contract No. MOST 104-2221-E-224-023.

Table 2. Comparison of security issues

\begin{tabular}{cccccccc}
\hline & $\begin{array}{c}\text { Zhu-Ma } \\
\text { scheme [12] }\end{array}$ & $\begin{array}{c}\text { LHL scheme } \\
{[\mathbf{5}]}\end{array}$ & $\begin{array}{c}\text { WLT scheme } \\
{[\mathbf{8}]}\end{array}$ & $\begin{array}{c}\text { Lee-Kwon } \\
\text { scheme [6] }\end{array}$ & $\begin{array}{c}\text { HMZCB } \\
\text { scheme [3] }\end{array}$ & $\begin{array}{c}\text { KWC scheme } \\
{[\mathbf{4}]}\end{array}$ & Ours \\
\hline$P_{1}$ & No & No & No & Yes & No & Yes & Yes \\
\hline$P_{2}$ & Yes & No & No & No & Yes & Yes & Yes \\
\hline$P_{3}$ & No & No & Yes & Yes & Yes & Yes & Yes \\
\hline$P_{4}$ & Yes & Yes & Yes & Yes & Yes & Yes & Yes \\
\hline$P_{5}$ & No & No & No & Yes & No & Yes & Yes \\
\hline$P_{6}$ & Yes & Yes & Yes & No & No & No & No \\
\hline$P_{7}$ & No & No & No & No & No & No & Yes \\
\hline$P_{1}:$ Achieve Anonymity; $P_{2}:$ Prevent Man-in-the-middle attack; $P_{3}:$ Secrecy of session key; $P_{4}:$ Prevention of Replay attack; \\
\hline \multicolumn{7}{c}{}
\end{tabular}

\section{References}

[1] K. Chain, W.C. Kuo. A new digital signature scheme based on chaotic maps. Nonlinear Dynamics, 2013, Vol.74, No.4, 1003-1012.

[2] C. Guo, C.C. Chang, C.Y. Sun. Chaotic maps-based mutual authentication and key agreement using smart cards for wireless communications. Journal of Information Hiding and Multimedia Signal Processing, 2013, Vol.4, No.2, 99-109.

[3] D. He, M. Ma, Y. Zhang, C. Chen, J. Bu. A strong user authentication scheme with smart cards for wireless communications. Computer Communications, 2011, Vol.34, No.3, 367-374.

[4] W.C. Kuo, H.J. Wei, J.C. Cheng. An efficient and secure anonymous mobility network authentication scheme. Journal of Information Security and Applications, 2014, Vol.19, No.1, 18-29.

[5] C.C. Lee, M.S. Hwang, I.E. Liao. Security enhancement on a new authentication scheme with anonymity for wireless environments. IEEE Trans. Industrial Electron., 2006, Vol.53, No.5, 1683-1687.

[6] J.H. Lee, T.Y. Kwon. Secure authentication scheme with improved anonymity for wireless environments. IEICE Trans. Commun., 2011, Vol.E94-B, No.2, 554557.

[7] J.S. Lee, J. Chang, D. Lee. Security flaw of authentication scheme with anonymity for wireless communications. IEEE Communications Letters, 2009, Vol.13, No.5, 292-293.

[8] C.C. Wu, W.B. Lee, W.J. Tsaur. A secure authentication scheme with anonymity for wireless communications. IEEE Communication Letters, 2008, Vol.12, No.10, 722-723.

[9] D. Xiao, X. Liao, S. Deng. One-way hash function construction based on the chaotic map with changeable parameter. Chaos Solitons \& Fractals, 2005, Vol.24, No.1, 65-71.

[10] D. Xiao, X. Liao, S. Deng. A novel key agreement protocol based on chaotic maps. Information Sciences, 2007, Vol.177, No.4, 1136-1142.

[11] P. Zeng, Z. Cao, K. Choo, S. Wang. On the anonymity of some authentication schemes for wireless communications. IEEE Communications Letters, 2009, Vol.13, No.3, 170-171.

[12] J. Zhu, J. Ma. A new authentication scheme with anonymity for wireless environments. IEEE Trans. Consumer Electron., 2004, Vol.50, No.1, 231-235.

Received December 2014 